\title{
Development of a Discriminating Dissolution Method for Immediate-Release Soft Gelatin Capsules Containing a BCS Class II Compound
}

\author{
Festo Damian ${ }^{1}$, Mohammad Harati², Vinayak Pathak², Jeff Schwartzenhauer², \\ Doug Durham², Vince Quiquero², Owen Van Cauwenberghe ${ }^{1,2}$, and Shawn D. Wettig ${ }^{1,3, *}$ \\ ${ }^{1}$ School of Pharmacy, University of Waterloo, 200 University Ave. W, Waterloo, ON N2L 3G1, Canada \\ ${ }^{2}$ Accucaps Industries Ltd., 2125 Ambassador Dr., Windsor, ON N9C 3R5, Canada \\ ${ }^{3}$ Waterloo Institute for Nanotechnology, University of Waterloo, 200 University Ave. W, Waterloo, ON N2L 3G1, Canada
}

\section{ABSTRACT}

The purpose of this study was to develop a robust dissolution procedure for liquid-filled, soft gelatin capsules (SGCs) that can distinguish small but real changes in drug product formulation. SGCs were manufactured using different ratios of fill formulations. The formulations were evaluated by performing dissolution testing on fresh capsules and capsules that were aged at different time points and conditions. USP Apparatus 1 (basket) and 2 (paddle) at rotating speeds of 50, 75 , or $100 \mathrm{rpm}$ were used to evaluate the release characteristics of the formulations. The model-independent method using difference factor $\left(f_{1}\right)$ and similarity factor $\left(f_{2}\right)$ was employed to compare dissolution profiles. Dissolution data showed that fill material characteristics played a major role in controlling the rate of dissolution of our drug products. The basket increased the discriminatory power of the dissolution process regardless of speed. We observed that the paddle rotation speed may impact discrimination power but not the ability of the method to discriminate. The basket was successful for the quality assessment and characterization of formulation changes of our drug products. Through understanding of drug product characteristics and evaluating parameters of dissolution testing, a methodology can be established to enable batch-to-batch evaluation.

KEYWORDS: Soft gelatin capsules; dissolution; stability; loratadine; poorly soluble drugs.

\section{INTRODUCTION}

ormulation of drugs with poor aqueous solubility poses several challenges, since solubility dictates the amount of drug dissolved and thus bioavailability. Compounds with low aqueous solubility will undergo rate-limited absorption in the gastrointestinal tract (GIT) resulting in incomplete absorption of the drug (1). Encapsulation of these drug products into soft gelatin capsules (SGCs) is one of the various approaches that have been employed to improve the biopharmaceutical performance of poorly soluble drugs $(1,2)$. Recently, SGC encapsulation has gained much interest because the majority of new chemical entities are poorly water soluble (3-5).

When SGCs are introduced into biological media, normally they undergo several intermediate steps that are crucial for product development (i.e., rupture of the soft gelatin shell, release and dispersion of the capsulefill materials, and dissolution of the active ingredient), as shown in Figure 1. These steps are followed by drug

*Corresponding author. absorption, biodistribution, and elimination leading to a biological response, resulting in overall therapeutic benefits (6). Dissolution testing is used throughout the course of the product development lifecycle since it helps

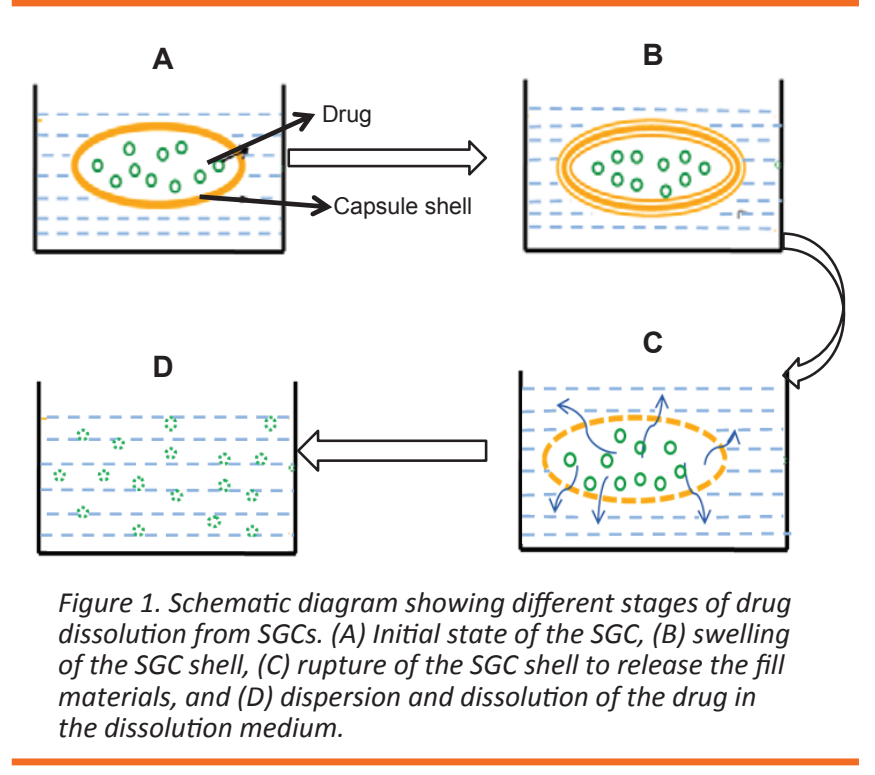


in developing and evaluating new drug formulations by studying the rate of drug release from dosage forms, evaluating the stability of these formulations, monitoring drug product consistency, assessing formulation changes, and establishing in vitro-in vivo correlation (IVIVC) (7). SGCs can present challenges for developing a robust dissolution testing method, particularly when filled with hydrophobic carrier materials. The majority of methods employed are typically designed for solid tablet dosage forms, which disintegrate and disperse uniformly. Oily liquid fill materials in SGCs lead to oil droplets suspended and dispersed in the dissolution medium that eventually may phase-separate and form layers above the aqueous medium. The existence of oil droplets may hinder the release of the drug or cause sampling problems leading to high variability in measured drug release. Differences in release patterns can also be caused by variability of the dispersion of the fill material in dissolution media and locations of the test capsule or sampling probe. Likewise, the behavior of emulsified or solubilized formulations during dissolution testing can present significant problems for monitoring of the release profile.

From the discussion above, it can be seen that it is necessary to develop a dissolution strategy specific to SGCs and in particular, to develop a method that has the capability to detect changes in the drug product formulation and potential performance. Demonstrating the discriminatory power of the dissolution method is both challenging and important, particularly in monitoring the active pharmaceutical ingredient (API) or formulation parameters where subtle changes may affect biopharmaceutical product performance. The discriminatory power of the dissolution method is the method's ability to detect changes in the drug product ( 8 , 9).

The model compound that was used in this study is a poorly soluble drug, loratadine. It is a derivative of azatadine and a second-generation histamine $\mathrm{H} 1$ receptor antagonist used in the treatment of allergic rhinitis and urticaria. Unlike most classical antihistamines, it lacks central nervous system depressing effects such as drowsiness (10). Loratadine is a Biopharmaceutics Classification System (BCS) Class II drug, with dissolutionor solubility-limited absorption (11). It is a weak base, with a p $K_{\mathrm{a}}$ value at $25^{\circ} \mathrm{C}$ reported to be $4.85-6.00$ (10-12). The $\mathrm{pH}$-dependent solubility of loratadine has been described at different $\mathrm{pH}$ ranges by Frizon et al. (13) and Khan et al. (11) and was $484.27 \mu \mathrm{g} / \mathrm{mL}, 4.32 \mu \mathrm{g} / \mathrm{mL}$, and $3.16 \mu \mathrm{g} / \mathrm{mL}$ at $\mathrm{pH} 20.1 \mathrm{~N} \mathrm{HCl}, \mathrm{pH} 6.8$ phosphate buffer, and pH 12.5 tris(hydroxymethyl)aminomethane buffer, respectively.
The objective of this study was to develop a dissolution procedure that is robust and able to distinguish between SGCs that contain different fill formulations. The data obtained should be able to reflect the product change but not method variation.

\section{MATERIALS AND METHODS Materials}

Loratadine SGCs and loratadine reference standard were obtained from Accucaps Industries Limited (Windsor, Canada). Pepsin from porcine gastric mucosa was purchased from Sigma-Aldrich (St. Louis, MO, USA). PVDF (polyvinylidene fluoride) membrane syringe filters (0.2$\mu \mathrm{m}$ pore size, $25-\mathrm{mm}$ diameter) were purchased from GE Healthcare Ltd (Buckinghamshire, UK). Sodium phosphate dibasic, sodium phosphate monobasic monohydrate, sodium lauryl sulfate (SDS), hydrochloric acid, trifluoroacetic acid, HPLC grade acetonitrile, methanol, and water were purchased from Sigma-Aldrich (St. Louis, MO, USA).

\section{Preparation of SGCs}

All SGCs were prepared at Accucaps Industries Limited (Windsor, Canada). Different ratios of medium-chain monoglycerides (MCM), medium-chain triglycerides (MCT), surfactants, solvents, hydrophilic carriers, active ingredient, and dispersant were thoroughly mixed stepwise until a homogenous mixture was formed. The solubilizers and the carrier materials were heated to temperatures between 55 and $65{ }^{\circ} \mathrm{C}$. Loratadine was then added while mixing continuously for $30 \mathrm{~min}$. This was followed by the addition of surfactant, and finally the solvent and the dispersants were added while continuously mixing for an additional $30 \mathrm{~min}$. The solutions were cooled by circulating cold water through the jacketed tank until they reached $32{ }^{\circ} \mathrm{C}$. The solutions were then blanketed with nitrogen then encapsulated using a soft gelatin encapsulation filling machine followed by air drying until the capsules reached the hardness of not less than $8.5 \mathrm{~N}$ (the ideal hardness is between 8.5 and 10 $\mathrm{N}$; SGCs with hardness greater than $10 \mathrm{~N}$ would be brittle). All other parameters, such as weight uniformity, content uniformity, and residual water content were controlled at this stage. The composition of each formulation is shown in Table 1. The shell composition was consistent across the different fill formulations. Some samples were kept at $25^{\circ} \mathrm{C}$ (long-term stability) while others at accelerated conditions (i.e., $40^{\circ} \mathrm{C} / 75 \% \mathrm{RH}$ ) for six months.

\section{Dissolution Test Conditions}

Dissolution testing was evaluated using Vision Classic 6 Dissolution Testers (Hanson Co., Chatsworth, CA, USA). 
Table 1. Composition of Formulations Used in the Dissolution Study

\begin{tabular}{|l|c|c|c|}
\hline \multirow{2}{*}{ Fill material } & Form. 1 & Form. 2 & Form. 3 \\
\cline { 2 - 4 } & $\%(w / w)$ & $\%(w / w)$ & $\%(w / w)$ \\
\hline Medium chain triglycerides (MCT) & 41.18 & 20.62 & 0 \\
\hline Mono- and diglycerides (MCM) & 41.18 & 61.75 & 82.38 \\
\hline API USP & 6.25 & 6.25 & 6.25 \\
\hline Polysorbate 80 NF/EP & 3.125 & 3.125 & 3.125 \\
\hline Purified water, NF & 2 & 2 & 2 \\
\hline Povidone K12, PF USP & 6.25 & 6.25 & 6.25 \\
\hline Total percentage & 100 & 100 & 100 \\
\hline Ratio of MCM to MCT & $50: 50$ & $75: 25$ & $100: 0$ \\
\hline
\end{tabular}

The influence of rotation speed, dissolution medium, and type of dissolution apparatus was evaluated. USP Apparatus 1 (basket) and 2 (paddle) were used at rotation speeds of 50,75 , or $100 \mathrm{rpm}$. USP baskets (40-mesh, 316 SS) were used for USP Apparatus 1 . The volume of the dissolution medium was $900 \mathrm{~mL}$ in each dissolution vessel $(0.1 \mathrm{~N} \mathrm{HCl}$ in purified water); $900 \mathrm{~mL}$ is a standard volume commonly used in dissolution testing and fulfilled the sink conditions of the experiments. The medium was deaerated by purging with helium for 5 min and then maintained at $37 \pm 0.5{ }^{\circ} \mathrm{C}$ during the dissolution process. Dissolution samples were collected manually at specified time intervals (i.e., 10, 15, 20, 30, 45, and $60 \mathrm{~min}$ ), immediately filtered through $0.2-\mu \mathrm{m}$ PVDF membrane filters into HPLC vials, then analyzed using a validated HPLC method. Six SGCs were evaluated for each drug product tested for each condition. The amount of loratadine in the test samples was calculated as a percentage dissolved from the measured peak areas of the test samples compared with the peak areas of the standard loratadine using the following equation:

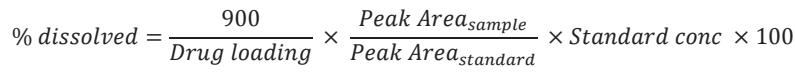

\section{HPLC Analysis}

Dissolution samples were analyzed using a validated HPLC method on a Waters Alliance e2695 HPLC system (YMCPack Pro C18 RS column; $80 \AA$, $150 \times 4.6 \mathrm{~mm}, 3 \mu \mathrm{m}$ ) with a Waters 2489 UV-vis detector. UV signals were monitored at $272 \mathrm{~nm}$, and peaks were integrated using Empower Software. The column temperature was set at $50^{\circ} \mathrm{C}$ with an injection volume of $50 \mu \mathrm{L}$. Mobile phase A consisted of $0.1 \%$ TFA in water, and mobile phase $B$ was acetonitrile. A 10-min gradient method was utilized, which went from $65: 35 \mathrm{~A} / \mathrm{B}$ to $2: 98 \mathrm{~A} / \mathrm{B}$ in $8 \mathrm{~min}$ and then equilibrated at
65:35 A/B for 2 min. At these conditions, the retention time of loratadine was approximately $4.1 \mathrm{~min}$. The sample run was always preceded and ended by a set of loratadine standards. After every 10 samples, one of the standard solutions was injected to ensure that the instrument did not drift during the run.

\section{Dissolution Data Evaluation}

A plot of the dissolution profile for each run was compared to determine the most discriminating dissolution conditions to be used for further experiments. A modelindependent approach and Student's $t$-test were used to evaluate the dissolution profiles of the formulations. The model-independent approach (14) includes the difference factor $\left(f_{1}\right)$ and the similarity factor $\left(f_{2}\right)$. The $f_{1}$ factor measures the percentage error between two curves over all time points:

$$
f_{1}=\left\{\left[\sum_{t=1}^{n}\left|R_{t}-T_{t}\right|\right] /\left[\sum_{t=1}^{n} R_{t}\right]\right\} \times 100
$$

where $n$ is the number of time points and $R_{\mathrm{t}}$ and $T_{\mathrm{t}}$ are the percentages of the reference and test product dissolved, respectively, at each time point. The percentage error is zero when the test and drug reference profiles are identical and increases proportionally with the dissimilarity between the two dissolution profiles. The similarity factor $\left(f_{2}\right)$ is a logarithmic transformation of the sum-squared error of differences of drug percentage dissolved between the test and the reference products over all time points:

$$
f_{2}=50 \cdot \log \left\{\left[1+\frac{1}{n} \sum_{t=1}^{n}\left(R_{t}-T_{t}\right)^{2}\right]^{-0.5} \times 100\right\}
$$

\section{RESULTS AND DISCUSSION}

\section{Dissolution Method Development and Validation}

Before starting the dissolution studies, we first validated the analytical (HPLC) method. The optimized method was linear $\left(r^{2}=0.9999\right)$, accurate (RSD $\left.=0.6 \%\right)$, and precise (RSD between 1.7 and 1.9\% for control and stressed samples, respectively), thus it was suitable for detection and quantification of loratadine.

\section{Dissolution Studies}

To develop a successfully robust dissolution test method for SGCs, a good understanding of the dissolution of the drug in different media must be established. To establish a suitable medium, several different dissolution media should be evaluated to identify the one that achieves appropriate sink conditions. The first step in these 
experiments was to select a dissolution medium suitable for dissolution of drug product. The FDA guidance on dissolution testing (14) recommends comparing the dissolution profiles of such drug products in several dissolution media in the $\mathrm{pH}$ range of 1-6.8. The initial dissolution tests were carried out under the following conditions: $0.1 \mathrm{~N} \mathrm{HCl}, 0.1 \mathrm{M} \mathrm{pH} 6.2$ phosphate buffer, and $0.1 \mathrm{M} \mathrm{pH} 8.2$ phosphate buffer. Sometimes the dissolution of poorly soluble drugs requires dissolution media that are different from those normally used for water-soluble drugs. One technique that is useful in the dissolution of such drugs is the incorporation of a small amount of surfactant in the dissolution medium $(15,16)$. The use of surfactants in dissolution systems may be considered as physiologically meaningful because of the presence of natural surfactants such as bile salts and bile acids in the gastrointestinal tract (17). The ability of surfactants to accelerate the in vitro dissolution of poorly soluble drugs has been attributed mainly to increases in wetting and the micellar solubilization process (18). Based on these considerations, the influence of surfactant on the dissolution properties of the drug products was evaluated by adding sodium lauryl sulfate to the dissolution medium (i.e., $0.1 \% \mathrm{SLS}$ and $0.5 \% \mathrm{SLS}$ in $0.1 \mathrm{~N} \mathrm{HCl}$ ). The preliminary solubility data showed that loratadine is more soluble in SLS than in polysorbate 80; hence, SLS was only used in the preliminary dissolution studies. This preliminary work was done using a paddle, stirring at 50 and $100 \mathrm{rpm}$.

The data obtained show that $0.1 \mathrm{M}$ phosphate buffer $\mathrm{pH}$ 6.2 and 8.2 failed to dissolve the API because no loratadine was detected in the dissolution medium. This shows that the solubility of the drug in the dissolution medium plays a major role in controlling the release properties of API from SGCs. This is not surprising because loratadine is a weak base with a $p K_{a}$ of 5.25 , and its aqueous solubility decreases with increasing $\mathrm{pH}$ (19). This finding ruled out both of the phosphate buffers as appropriate dissolution media for loratadine SGCs drug products. The next step in this set of experiments was to evaluate the dissolution of the drug product in $0.1 \mathrm{~N} \mathrm{HCl}$ and $0.1 \% \mathrm{SLS}$ in $0.1 \mathrm{~N} \mathrm{HCl}$ at 50 and $100 \mathrm{rpm}$ using the paddle method. The dissolution profiles in both of these media at $100 \mathrm{rpm}$ are similar. Both experienced a very small spike in the profile near the beginning of the run (the 5- and 10-min points), followed by smooth curves near the $100 \%$ level until the end of the dissolution run. Variations in the dissolution profiles were common at lower time points in contrast to the end of the curve, where the profiles were smooth. Higher rotation speeds caused a burst drug release reaching $100 \%$ within 5 to $10 \mathrm{~min}$, and this potentially masks the differences between the batches. Similar observations have been reported (20). The spikes in the dissolution profiles were present regardless of which of the two media was used at the lower speed. However, the dissolution media containing SLS showed low drug content release, and the peaks of the drug were distorted. A similar observation of a decrease in the dissolution of gelatin capsules with sodium lauryl sulfate at lower $\mathrm{pH}$ has been reported (21). Peak distortion with no improvement in dissolution was also observed for the dissolution of SGCs using $0.5 \%$ SLS in $0.1 \mathrm{~N} \mathrm{HCl}$. As a result, the dissolution medium that was selected for future experiments was $0.1 \mathrm{~N} \mathrm{HCl}$ in purified water.

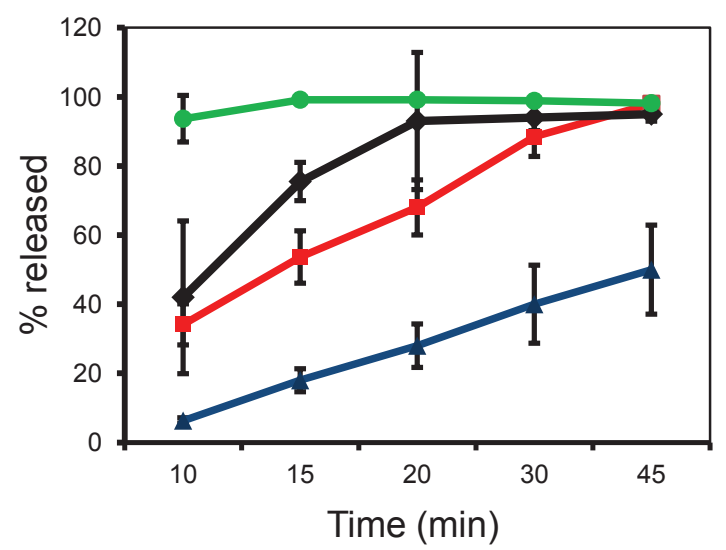

Figure 2. Dissolution profiles of loratadine SGCs using the basket and paddle methods, $0.1 \mathrm{~N} \mathrm{HCl}$ in purified water as dissolution medium: (০) $100 \mathrm{rpm}$, paddle; ( $\$ 50 \mathrm{rpm}$, paddle; ( $\square) 100 \mathrm{rpm}$, basket; and (\) 50 rpm, basket. Each data point represents the average \pm standard deviation $(n=6)$.

Selection of the correct dissolution apparatus is another critical step for SGC evaluation because mixing efficiency of fill materials with the dissolution medium is greatly influenced by the agitation hydrodynamics, especially by variables such as rotation speed and flow rate (22). The paddle method is commonly used for evaluating dissolution properties of SGCs. One major problem with use of the paddle is that SGCs, which can have a density less than that of water, can float to the surface. In this study, the capsules remained submerged (i.e., density greater than water), and sinkers were not required. The cumulative percentage of loratadine released using this method is presented in Figure 2. The release was evaluated using $0.1 \mathrm{~N} \mathrm{HCl}$ in purified water at rotation speeds of 50 and $100 \mathrm{rpm}$. The data obtained using the paddle at 100 rpm show $100 \%$ burst release of the drug within 15 min. On the other hand, a gradual increase in drug release that was greater than $40 \%$ after 10 min was observed at a speed of $50 \mathrm{rpm}$. Large variations in loratadine release were observed using this method, particularly 
at lower time points. Based on these results, the paddle is not the best method of discrimination because at 100 rpm, drug release reached $100 \%$ within a few minutes and no calculations could be made to differentiate the dissolution profiles, while lower dissolution speeds resulted in data with variable dissolution profiles. The variations at the lower time points were attributed to the lipophilic materials forming oil globules that could be easily collected during sampling. This resulted from the oil-fill materials being released from the capsule but not fully dissipated in the medium. These oil droplets can be sampled unintentionally when withdrawing samples from the dissolution vessels. The oil globules have high drug concentration, especially at the lower time points, resulting in the large variability observed.

To find a method that is more discriminating and less variable, the same drug products were evaluated using the basket. This method has the advantage of enclosing SGCs within a mesh basket, preventing them from floating in the medium. However, the soft gelatin shell may disintegrate into a soft and sticky mass that can clog the basket mesh, generating highly variable results. If the fill material is hydrophobic, it may not be sufficiently dispersed into droplets that are fine enough to pass through the basket mesh, resulting in a low percentage release. For example, Pillay et al. (23) used a rotating basket to conduct dissolution studies of lipid-filled SGCs, where most of the viscous oily vehicle remained entrapped within the basket after $6 \mathrm{~h}$ of dissolution. The basket showed a gradual release of the drug even at a 100-rpm stirring speed (Figure 2). The cumulative percentage drug released using the basket at $100 \mathrm{rpm}$ was generally lower than those collected using the paddle method at $50 \mathrm{rpm}$. Additionally, the basket showed less variation at lower time points and generally, this method was more discriminating compared with the paddle method. Despite the potential for the basket to interfere with dispersion of the oil droplets (described above), in this case, the basket may aid in dissipating the oil droplets more uniformly, minimizing the variability observed using the paddle method. Hence, the slower profile afforded by the basket reduced variations, so in this case, there was a better view of what was occurring throughout the dissolution process with less background noise. The influence of rotation speed on the dissolution of loratadine SGCs using the basket was evaluated in greater detail (Figure 3). The analysis of variance shows significant differences among the results obtained at 50, 75, and $100 \mathrm{rpm}(p<0.05)$, with the cumulative percentage drug release increasing as a function of rotation speed.

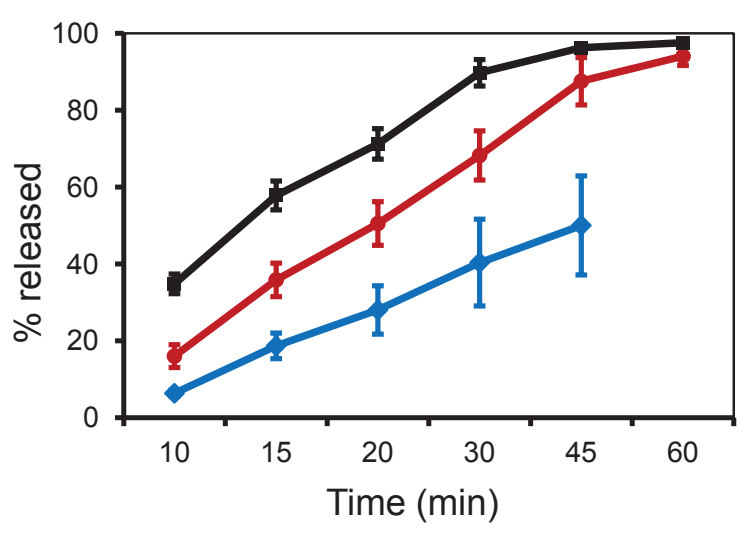

Figure 3. Dissolution profiles of loratadine SGCs using the basket, $0.1 \mathrm{~N} \mathrm{HCl}$ in purified water as dissolution medium, and different rotation speeds: (-) $100 \mathrm{rpm}$; (๑) $75 \mathrm{rpm}$; and (४) $50 \mathrm{rpm}$. Each data point represents the average \pm standard deviation $(n=6)$.

Since the basket showed the best results, further experiments were done using this method. Developing a discriminating dissolution test requires special considerations and knowledge of SGC fill material properties and factors influencing them. The SGC dissolution involves three stages including rupture of the shell, release and dispersion of the fill material, and dissolution of the active ingredient(s) in the dissolution medium. The most common approach to assess the discriminatory ability of the method is to test formulations with different forms, physical properties, drug product composition, and manufacturing conditions or stability conditions $(8,14)$. Apart from the capsule shells, the other main factor affecting the dissolution of SGCs is the composition of the fill materials. Types of fill materials may include lipophilic liquids or oils, hydrophilic liquids, self-emulsifying oils, microemulsions, nanoemulsions, and suspensions. Therefore, it is very important to have a dissolution method that is able to reflect the product change but is not susceptible to method variations. In the present study, the dissolution profiles of new formulations were evaluated by varying the ratios of the fill materials. Formulations with high ratios of mediumchain triglycerides had higher dissolution rates than those with high ratios of medium-chain monoglycerides (Figure 4). Typical acceptance criteria for the amount of drug dissolved should be not less than $85 \%$ in 60 min for fast-dissolving drug products or less for a routine quality control test for batch-to-batch uniformity (14). In the present study, the dissolution method was discriminatory, and the formulations released greater that $85 \%$ of API within 30 min (Figure 4), hence the acceptance criterion was met. The data collected using the basket demonstrate that this method is more discriminating and dissolution 
profiles correlate very well with the fill composition of the drug product. In vitro dissolution profiles are commonly used to compare the in vitro release of two drug products or formulations. In this respect, the dissolution profiles of the formulations were compared using the similarity factor $\left(f_{2}\right)$ and difference factor $\left(f_{1}\right)(14,24)$. Two dissolution profiles are considered to be similar when the $f_{2}$ value is greater than 50 or when the mean percentage difference between two curves has an $f_{1}$ less than 15 (24). Data presented in Table 2 show that $f_{2}$ values of three formulations are less than 50 , indicating that the release characteristics of these formulations are not similar. On the other hand, the difference factor $\left(f_{1}\right)$ calculated from the percentage difference between the two dissolution profiles at each time point shows values greater than 50 . Drug products that are similar have difference factors less than 15 , hence these findings support the results from similarity factor calculations that the dissolution profiles of our drug products are not similar. These data show that the dissolution properties of the drug products were influenced by the fill-material characteristics, whereby formulations with more MCT had higher dissolution rates than those with $100 \% \mathrm{MCM}$. Hence, fill-material characteristics play a major role in controlling the dissolution rate of SGCs and hence dissolution media selection (Figure 4 and Table 2).

Table 2. Comparison of Dissolution Profiles of New Drug Products with Reference Drug Product (RDP) Using Difference Factor $\left(f_{1}\right)$ and Similarity Factor $\left(f_{2}\right)$

\begin{tabular}{|c|c|c|c|c|}
\hline Drug product & MCM/MCT ratio & $\%$ released at 20 min & $f_{1}$ & $f_{2}$ \\
\hline Form. \# 1 & $50: 50$ & $104.8 \pm 2.3$ & 134 & 10 \\
\hline Form. \# 2 & $75: 25$ & $92.8 \pm 2.8$ & 91 & 18 \\
\hline Form. \# 3 & $100: 0$ & $61.0 \pm 14.1$ & 53 & 30 \\
\hline RDP & - & $50.1 \pm 5.7$ & - & - \\
\hline
\end{tabular}

\section{Stability Studies}

To evaluate the effect of storage conditions on the dissolution properties of the drug products under investigation, the dissolution behavior of stressed and stored capsules was studied. Significant changes in the drug dissolution characteristics observed over longterm storage of the dosage form indicate that functional changes in the drug product that may compromise in vivo performance are occurring. Figure 5 shows representative dissolution profiles of loratadine formulations stored at 25 and $40{ }^{\circ} \mathrm{C}$ for 6 months. The data show that the drug released from SGCs stored under accelerated conditions was less than $30 \%$ after $30 \mathrm{~min}$. On the other hand, drug products that were stored at $25{ }^{\circ} \mathrm{C}$ maintained their release properties with $80-100 \%$ of loratadine being

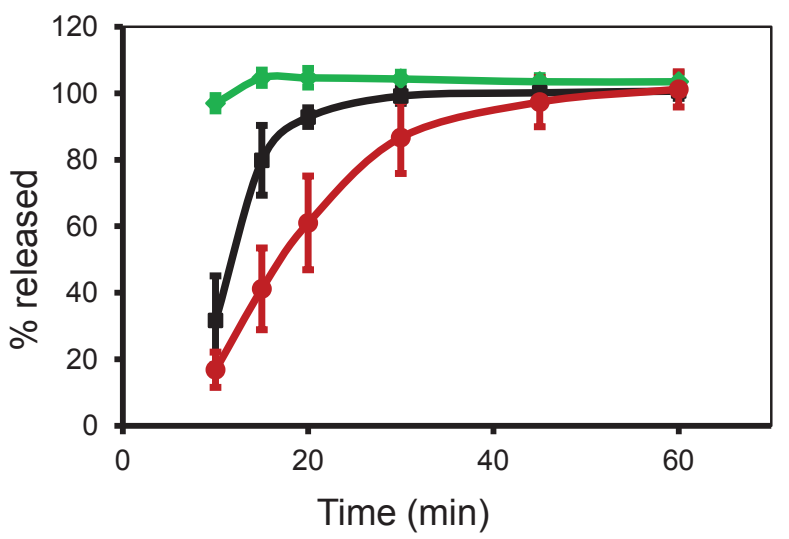

Figure 4. Dissolution profiles of loratadine drug products formulated using different concentrations of the fill materials using the basket at $75 \mathrm{rpm}$ in $0.1 \mathrm{~N} \mathrm{HCl}$ purified water: $(\diamond)$ Formulation 1 ;

(匹) Formulation 2; and (๑) Formulation 3. Each data point represents the average \pm standard deviation $(n=6)$.

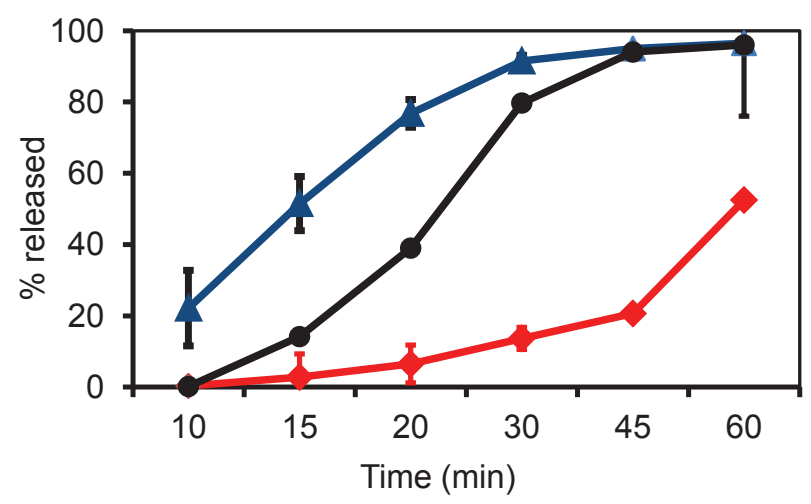

Figure 5. Dissolution profiles of SGCs stored at long-term storage conditions compared with those stored at accelerated conditions:

$(\Delta)$ long-term storage conditions $\left(25^{\circ} \mathrm{C}\right),(\Delta)$ accelerated conditions $\left(40{ }^{\circ} \mathrm{C} / 75 \% \mathrm{RH}\right)$ without enzyme, and (๑) accelerated conditions $\left(40{ }^{\circ} \mathrm{C} / 75 \% \mathrm{RH}\right)$ with enzyme. Each data point represents the average \pm standard deviation $(n=6)$.

released after $30 \mathrm{~min}$. The reduction in dissolution rate for capsules stored at the accelerated conditions suggests that there is a change in the physical structure of the SGCs. Interestingly, visual observation did not show any evidence of clogging or pellicle formation for capsules stored under the accelerated conditions. However, the SGCS possibly absorbed moisture, and this altered the shell integrity at higher temperature, thereby impacting the overall integrity of the capsule shell.

Several reports have hypothesized that the reduced dissolution of active ingredients from gelatin-based capsules results from cross-linking of the gelatin. Chafetz et al. (25) reported that a considerable decrease in the dissolution rate of the drug gemifibrozil was observed from SGC capsule formulations that were stored at 37 
${ }^{\circ} \mathrm{C}$ and $45{ }^{\circ} \mathrm{C} / 80 \% \mathrm{RH}$ for up to a 3-month period (25). Their data show that capsules containing Polysorbate 80 showed film formation after 1 month at $37{ }^{\circ} \mathrm{C} / 80 \%$ $\mathrm{RH}$, and the dissolution was lower after storage at these conditions. The decrease in capsule dissolution was due to formaldehyde formation from Polysorbate 80 autooxidation (25). Likewise, in another study (26), nifedipine SGCs failed the dissolution test using the paddle as a result of pellicle formation upon storage for 208 days at $25{ }^{\circ} \mathrm{C} / 60 \% \mathrm{RH}$ and $40{ }^{\circ} \mathrm{C} / 75 \% \mathrm{RH}$. However, despite the low in vitro release properties, several studies have shown that gelatin capsules with low dissolution rates are bioequivalent to those with good dissolution profiles (27). Therefore, poor in vitro dissolution properties during storage may not reflect poor performance in vivo. It has also been shown that gelatin capsules tested in dissolution media containing enzymes may not exhibit the slow dissolution observed for drug products stored under accelerated conditions $(28,29)$. Pepsin and pancreatin are present in the gastrointestinal tract and are recommended to be included in the dissolution media as per FDA guidance on dissolution testing (14), especially when soft and hard gelatin capsules show a decrease in dissolution rate over time without enzymes. Digestive enzymes play a key role in disrupting gelatin cross-linking, promoting rupture of the cross-linked gelatin shell, and enhancing the dissolution rate of the drug. Pepsin can act suitably in the $\mathrm{pH}$ range between 1 and 5 (30), while pancreatin (containing a mixture of enzymes including trypsin, amylase, lipase, and protease) is functional between $\mathrm{pH} 6$ and 8. To assess the effect of digestive enzymes on the dissolution properties of the drug products, the effect of pepsin on the dissolution properties of the accelerated stored SGCs was evaluated. Figure 5 clearly shows the enhancement of loratadine release using pepsin in the release medium. However, the release of drug was slightly lower compared with the original dissolution profile. The dissolution method developed was still discriminatory for the aged drug products, and the influence of GIT enzymes on the dissolution properties of these drug products was observed.

\section{CONCLUSIONS}

Validation results demonstrate that the dissolution method described in this work is accurate, precise, linear, robust, and specific. Dissolution rates obtained using the paddle method were faster (and highly variable at lower time points) than those obtained using the basket, which is attributed the higher agitation speed of the paddle method, resulting in burst release of the drug from the SGCs. On the other hand, the data collected using the basket demonstrate that this method is more discriminating and showed gradual release of the drug with less variation. The dissolution profiles correlate very well with the fill composition of the drug product. In general, we can conclude that the fill material characteristics play a major role in controlling the rate of dissolution for BCS Class II drug products. The basket increased the discriminatory power of the method regardless of speed; however, speed may impact discrimination power but not the ability to discriminate. Through understanding of product characteristics and evaluating parameters of dissolution testing, a methodology can be established to enable batch-to-batch evaluation. The dissolution method presented here can be used as a quality control test for lotatadine drug product.

\section{ACKNOWLEDGMENTS}

We gratefully acknowledge the School of Pharmacy at the University Waterloo for allowing access to the dissolution testing and HPLC instrumentation that are part of the undergraduate teaching laboratory.

\section{CONFLICT OF INTEREST}

This work was funded by Accucaps industries Ltd (Ontario, Canada). Accucaps was involved in the manufacturing of soft gelatin capsules and approved the manuscript before submission.

\section{REFERENCES}

1. Cole, E. T.; Cadé, D.; Benameur, H. Challenges and opportunities in the encapsulation of liquid and semi-solid formulations into capsules for oral administration. Adv. Drug Deliv. Rev. 2008, 60 (6), 747-756. DOI:10.1016/j. addr.2007.09.009.

2. Mainardes, R. M.; Silva, L. P. Drug Delivery Systems: Past, Present, and Future. Curr. Drug Targets 2004, 5 (5), 449455. DOI: $10.2174 / 1389450043345407$.

3. Anand, O.; Yu, L. X.; Conner, D. P.; Davit, B. M. Dissolution Testing for Generic Drugs: An FDA Perspective. AAPS J. 2011, 13 (3), 328-335. DOI: 10.1208/s12248-011-9272-y.

4. Shi, Y.; Porter, W.; Merdan, T.; Li, L. C. Recent advances in intravenous delivery of poorly water-soluble compounds. Expert Opin. Drug Delivery 2009, 6 (12), 1261-1282. DOI: 10.1517/17425240903307423.

5. Shah, V. P.; Amidon, G. L. A Theoretical Basis for a Biopharmaceutic Drug Classification: The Correlation of In Vitro Drug Product Dissolution and In Vivo Bioavailability, Pharm. Res. 12, 413-420, 1995-Backstory of BCS. AAPS J. 2014, 16 (5), 894-898. DOI: 10.1208/s12248-014-9620-9.

6. Camenisch, G.; Folkers, G.; van de Waterbeemd, H. Review of theoretical passive drug absorption models: Historical background, recent developments and limitations. Pharm. Acta Helv. 1996, 71 (5), 309-327. DOI: 10.1016/S00316865(96)00031-3. 
7. Brown, C. K.; Friedel, H. D.; Barker, A. R.; Buhse, L. F.; Keitel, S.; Cecil, T. L.; Kraemer, J.; Morris, J. M.; Reppas, C.; Stickelmeyer, M. P.; Yomota, C.; Shah, V. P. FIP/AAPS Joint Workshop Report: Dissolution/In Vitro Release Testing of Novel/Special Dosage Forms. AAPS PharmSciTech 2011, 12 (2), 782-794. DOI: 10.1208/s12249-011-9634-x.

8. Brown, C. K.; Chokshi, H. P.; Nicherson, B.; Reed, R. A.; Rohrs, B. R.; Shah, P. A. Acceptable Analytical Practices for Dissolution Testing of Poorly Soluble Compounds. Pharm. Technol. 2004, 28 (12), 56-65.

9. Limberg, J.; Potthast, H. Regulatory status on the role of in vitro dissolution testing in quality control and biopharmaceutics in Europe. Biopharm. Drug Dispos. 2013, 34 (5), 247-253. DOI: 10.1002/bdd.1844.

10. ter Laak, A. M.; Tsai, R. S.; Donné-Op den Kelder, G. M.; Carrupt, P.-A.; Testa, B.; Timmerman, H. Lipophilicity and hydrogen-bonding capacity of $\mathrm{H} 1$-antihistaminic agents in relation to their central sedative side-effects. Eur. J. Pharm. Sci. 1994, 2 (5-6), 373-384. DOI: 10.1016/09280987(94)00065-4.

11. Khan, M. Z. I.; Raušl, D.; Zanoški, R.; Zidar, S.; Mikulčić, J. H.; Krizmanić, L.; Eškinja, M.; Mildner, B.; Knežević, Z. Classification of Loratadine Based on the Biopharmaceutics Drug Classification Concept and Possible in Vitro-in Vivo Correlation. Biol. Pharm. Bull. 2004, 27 (10), 1630-1635. DOI: $10.1248 / \mathrm{bpb} .27 .1630$.

12. El-Hammadi, M.; Awad, N. Investigating the Use of Liquisolid Compacts Technique to Minimize the Influence of $\mathrm{pH}$ Variations on Loratadine Release. AAPS PharmSciTech 2012, 13 (1), 53-58. DOI: 10.1208/s12249011-9719-6.

13. Frizon, F.; de Oliveira Eloy, J.; Donaduzzi, C. M.; Mitsui, M. L.; Maldonado Marchetti, J. Dissolution rate enhancement of loratadine in polyvinylpyrrolidone $\mathrm{K}-30$ solid dispersions by solvent methods. Powder Technol. 2013, 235, 532-539. DOI:10.1016/j.powtec.2012.10.019.

14. Dissolution Testing of Immediate Release Solid Oral Dosage Forms; Guidance for Industry; U.S. Department of Health and Human Services, Food and Drug Administration, Center for Drug Evaluation and Research (CDER), U.S. Government Printing Office: Washington, DC, 1997.

15. Serajuddin, A. T. M.; Sheen, P.-C.; Augustine, M. A. Improved Dissolution of a Poorly Water-Soluble Drug from Solid Dispersions in Polyethylene Glycol: Polysorbate 80 Mixtures. J. Pharm. Sci. 1990, 79 (5), 463-464. DOI: 10.1002/jps.2600790524.

16. Serajuddin, A. T. M.; Mufson, D.; Bernstein, D. F.; Sheen, P.C.; Augustine, M. A. Effect of Vehicle Amphiphilicity on the Dissolution and Bioavailability of a Poorly Water-Soluble Drug from Solid Dispersions. J. Pharm. Sci. 1988, 77 (5), 414-417. DOI: 10.1002/jps.2600770512.

17. Dressman, J. B.; Vertzoni, M.; Goumas, K.; Reppas, C.
Estimating drug solubility in the gastrointestinal tract. Adv. Drug Deliv. Rev. 2007, 59 (7), 591-602. DOI: 10.1016/j. addr.2007.05.009.

18. Torchilin, V. P. Structure and design of polymeric surfactantbased drug delivery systems. J. Controlled Release 2001, 73 (2-3), 137-172. DOI: 10.1016/S0168-3659(01)00299-1

19. Popović, G.; Čakar, M.; Agbaba, D. Acid-base equilibria and solubility of loratadine and desloratadine in water and micellar media. J. Pharm. Biomed. Anal. 2009, 49 (1), 42-47. DOI: 10.1016/j.jpba.2008.09.043.

20. Gray, V.; Kelly, G.; Xia, M.; Butler, C.; Thomas, S.; Mayock, S. The Science of USP 1 and 2 Dissolution: Present Challenges and Future Relevance. Pharm. Res. 2009, 26 (6), 12891302. DOI: 10.1007/S11095-008-9822-X.

21. Zhao, F.; Malayev, V.; Rao, V.; Hussain, M. Effect of Sodium Lauryl Sulfate in Dissolution Media on Dissolution of Hard Gelatin Capsule Shells. Pharm. Res. 2004, 21 (1), 144-148. DOI: 10.1023/B:PHAM.0000012162.52419.b3.

22. Dokoumetzidis, A.; Macheras, P. A century of dissolution research: From Noyes and Whitney to the Biopharmaceutics Classification System. Int. J. Pharm. 2006, 321 (1-2), 1-11. DOI:10.1016/j.ijpharm.2006.07.011.

23. Pillay, V.; Fassihi, R. Evaluation and comparison of dissolution data derived from different modified release dosage forms: an alternative method. J. Controlled Release 1998, 55 (1), 45-55. DOI:10.1016/S0168-3659(98)00022-4.

24. Costa, P.; Sousa Lobo, J. M. Modeling and comparison of dissolution profiles. Eur. J. Pharm. Sci. 2001, 13 (2), 123133. DOI: 10.1016/S0928-0987(01)00095-1.

25. Chafetz, L.; Hong, W.-H.; Tsilifonis, D. C.; Taylor, A. K.; Philip, J. Decrease in the Rate of Capsule Dissolution Due to Formaldehyde from Polysorbate 80 Autoxidation. J. Pharm. Sci. 1984, 73 (8), 1186-1187. DOI: http://dx.doi. org/10.1002/jps.2600730846.

26. Bottom, C. B.; Clark, M.; Carstensen, J. T. Dissolution Testing of Soft Shell Capsules-Acetaminophen and Nifedipine. J. Pharm. Sci. 1997, 86 (9), 1057-1061. DOI: 10.1021/js960263k.

27. Murthy, K. S.; Reisch, R. G. J.; Fawzi, M. B. Dissolution Stability of Hard Shell Capsule Products. The Effect of Dissolution Test Conditions on In Vitro Drug Release. Pharm. Technol. 1989, 13 (6), 53-56.

28. <711> Dissolution. In The United States Pharmacopeia and National Formulary USP 38-NF 33; The United States Pharmacopeial Convention, Inc.: Rockville, MD, 2015.

29. Digenis, G. A.; Gold, T. B.; Shah, V. P. Cross-Linking of Gelatin Capsules and Its Relevance to Their in Vitro-in Vivo Performance. J. Pharm. Sci. 1994, 83 (7), 915-921. DOI: $10.1002 / j p s .2600830702$.

30. Piper, D. W.; Fenton, B. H. pH stability and activity curves of pepsin with special reference to their clinical importance. Gut 1965, 6 (5), 506-508. DOI:10.1136/gut.6.5.506. 